\title{
High prevalence of false positive SARS-CoV2 serology in a cohort of patients with liver autoimmune diseases
}

\author{
Maria Giulia Cornacchia, Moris Sangineto * ${ }^{\circledR}$, Rosanna Villani $\odot$, Francesco Cavallone, Giuseppe Di Gioia, \\ Paola Cicciomessere, Gaetano Serviddio
}

University Center for Liver Disease Research and Treatment (C.U.R.E.), Liver Unit, Department of Medical and Surgical Sciences, University of Foggia, Viale Pinto 1, 71121 Foggia, Puglia, Italy

${ }^{*}$ Correspondence: Moris Sangineto, University Center for Liver Disease Research and Treatment (C.U.R.E.), Department of Medical and Surgical Sciences, University of Foggia, 71121 Foggia, Puglia, Italy. moris.sangineto@unifg.it

Academic Editor: Amedeo Lonardo, Azienda Ospedaliero-Universitaria di Modena, Italy

Received: June 11, 2021 Accepted: August 5, 2021 Published: August 31, 2021

Cite this article: Cornacchia MG, Sangineto M, Villani R, Cavallone F, Di Gioia G, Cicciomessere P, et al. High prevalence of false positive SARS-CoV2 serology in a cohort of patients with liver autoimmune diseases. Explor Med. 2021;2:372-7. https://doi.org/10.37349/emed.2021.00055

\begin{abstract}
Aim: Monitoring the prevalence of severe acute respiratory syndrome coronavirus 2 (SARS-CoV2) immunization in patients with autoimmune diseases is of particular concern to understand their response to the infection and to the vaccine. In fact, the immunological disorder and the immunosuppressive therapies could affect the serological response. SARS-CoV2 serological tests potentially provide this information, although they were rapidly commercialized with internal verifications. Here, we analysed the seroprevalence to SARS-CoV2 in a cohort of patients with liver autoimmune diseases.

Methods: From May to December 2020, a cohort of patients affected by primary biliary cholangitis (PBC), autoimmune hepatitis (AIH) and PBC/AIH overlap syndrome were screened with (reverse transcription-polymerase chain reaction) RT-PCR of nasopharyngeal swabs, rapid antigenic test and chemiluminescent serological test during routine follow-up.

Results: The analysis of 42 patients was carried out: 18 (42.85\%) PBC, 12 (28.57\%) AIH and 12 (28.57\%) PBC/AIH overlap syndromes. Only 2 patients (4.76\%) resulted positive to the RNA, antigen and antibody detection tests, hence affected by SARS-CoV2 infection. 14 subjects out of 40 negative cases presented a positive serology for SARS-CoV2 antibodies, hence with a false positivity in the $35 \%$ of cases without infection. Among these, $6(42.86 \%)$ patients presented only immunoglobulin (Ig)M positivity, 6 (42.86\%) patients presented positivity for only IgG and $2(14.28 \%)$ patients were positive to both IgM and IgG. Notably, the presence of autoantibodies did not correlate with the serological false positivity, highlighting that there is no cross-reactivity with autoantibodies. The presence of polyclonal hypergammaglobulinemia did not interfere with the serological test as well. Interestingly, the patients with false positive serology showed higher levels of gamma-glutamyltransferase (GGT) and C-reactive protein (CRP).

Conclusions: Patients with liver autoimmune diseases present a high rate of false positive SARS-CoV2 serology. Therefore, new strategies are needed to study the serological response in this patient category.




\section{Keywords}

Chronic liver disease, liver autoimmune disease, SARS-CoV2, COVID-19, serology, autoantibodies

\section{Introduction}

The coronavirus disease 2019 (COVID-19), first reported in Wuhan (China) in December 2019, has become a global pandemic which caused more than 170 million cases and 3.5 million deaths to date [1]. The rapid and accurate diagnosis is crucial to stop its widespread. However, the diagnosis of COVID-19 is still a challenge as symptoms are not disease specific (e.g., fever, cough, and fatigue) [2]. The main available weapons to identify severe acute respiratory syndrome coronavirus 2 (SARS-CoV2) infection are the viral RNA detection by reverse transcription-polymerase chain reaction (RT-PCR) of nasopharyngeal swabs and the serum antibody detection [3]. The sensitivity of RT-PCR depends on the RNA amount in the sample, permitting to classify patients as "positive" or "negative" cases. Serological tests are useful to differentiate a recent infection from a possible long-lasting immunization as shown by the presence of immunoglobulin (Ig)M or IgG, respectively [4]. Moreover, reliable antibody detection methods are crucial for the identification of plasma donors, human mobility permission and development of vaccine strategies.

Monitoring the prevalence of SARS-CoV2 infection in patients with autoimmune diseases is of particular interest because of the immunological alterations and the immunosuppressive therapies characterising these subjects [5]. Accordingly, patients with autoantibodies are more susceptible to serious viral, bacterial and opportunistic fungal infections [6] and the serological response to the SARS-CoV2 infection and vaccines remains elusive in this particular patient subset. Most of the serological tests have been rapidly commercialized and independently verified. The specificity of this assay is usually determined by the cross-reactivity with other viral infections and very rarely is tested in patients with chronic inflammatory diseases [7].

The liver autoimmune diseases are immunological disorders characterised by hepatic inflammation with parenchymal and/or bile duct damage and autoantibodies production [8]. In this study, the SARS-CoV2 infection prevalence has been investigated in a cohort of patients with liver autoimmune diseases monitored at our Liver Unit, analysing the reliability of serological assay in this particular category.

\section{Methods}

From May to December 2020, during the first and the second epidemic waves, data of forty-two patients affected by primary biliary cholangitis (PBC), autoimmune hepatitis (AIH) and PBC/AIH overlap syndrome were collected during the routine follow-up at the University Center for Liver Disease Research and Treatment (C.U.R.E.), Department of Medical and Surgical Sciences, University of Foggia, Italy. The anamnestic exposure to COVID-19 cases and blood tests were collected. The detection of SARS-CoV2 infection was analysed with RT-PCR of nasopharyngeal swabs (Allplex ${ }^{\mathrm{TM}} 2019-\mathrm{nCoV}$, Assay Seegene Inc. Seoul, Korea), antigenic rapid test (Panbio $^{\mathrm{TM}}$, Abbott, Chicago, IL, USA), and quantitative chemiluminescent serological test (LIAISON ${ }^{\circledR}$ SARS-CoV2 IgM and S1/S2 IgG, DiaSorin, Saluggia, Italy) performed on LIAISON ${ }^{\circledR X L}$ (DiaSorin, Saluggia, Italy). Data were expressed in mean \pm standard deviation (SD). Differences between two groups were analysed with Student's $T$ test and chi-squared test for continuous variables and proportions, respectively. $P$ value $<0.05$ is considered as significant.

\section{Results}

Forty-two patients with autoimmune liver diseases were included in the study, whose baseline characteristics are shown in the Table 1. The study population was composed by $18(42.85 \%)$ PBC, 12 (28.57\%) AIH and 12 (28.57\%) PBC/AIH overlap syndromes. Two patients (4.76\%) resulted positive to RNA, antigen and antibody detection tests, hence affected by SARS-CoV2 infection. They showed only mild symptoms such as sore throat and asthenia, and positivity to both SARS-CoV2 IgM and IgG. Forty patients presented negative antigenic test and negative RT-PCR for RNA detection and denied symptoms and contact with potential SARS-CoV2 infected people in the previous three months. However, 14 subjects out of 40 negative cases presented a positive 
serology for SARS-CoV2 antibodies. These subjects were tested twice with RT-PCR of nasopharyngeal swab to confirm negativity. Therefore, the serological assay presented a false positive result in the $35 \%$ of cases without infection, and in particular, 6 (42.86\%) patients presented positivity for IgM only, 6 (42.86\%) patients presented positivity exclusively for IgG, and 2 (14.28\%) patients were positive to both IgM and IgG (Table 2). To further exclude the possibility of a past infection, the IgG-only positive subjects declared contacts in the previous 6 months with housemates only, who presented negative nasopharyngeal swabs and negative serology as well. Moreover, the serology of IgG-only positive patients was repeated monthly to exclude the typical decreasing titer trend of SARS-CoV2 antibodies.

Table 1. Baseline characteristics

\begin{tabular}{ll}
\hline Variable & $\boldsymbol{N}(\%)$ \\
\hline PBC & $18(42.85 \%)$ \\
AIH & $12(28.57 \%)$ \\
PBC/AlH overlap syndrome & $12(28.57 \%)$ \\
Positive RT-PCR & $2(4.76 \%)$ \\
Positive antigenic rapid test & $2(4.76 \%)$ \\
Positive serology & $2(4.76 \%)$ \\
False positive serology & $14(30.95 \%)$ \\
ANA positivity & $28(66.6 \%)$ \\
AMA positivity & $16(38.09 \%)$ \\
ANCA positivity & $4(9.52 \%)$ \\
ASMA positivity & $12(28.57 \%)$ \\
Anti-LKM positivity & $2(4.76 \%)$ \\
Variable & Mean $\pm \mathrm{SD}$ \\
Direct bilirubin (mg/dL) & $0.19 \pm 0.17$ \\
Indirect bilirubin (mg/dL) & $0.69 \pm 0.36$ \\
GOT (U/L) & $41.33 \pm 21.48$ \\
GPT (U/L) & $41.95 \pm 23.48$ \\
GGT (U/L) & $84.61 \pm 85.45$ \\
ALP (U/L) & $124.14 \pm 58.67$ \\
CRP (mg/L) & $4.06 \pm 6.08$ \\
ESR (mm/h) & $26.42 \pm 21.61$ \\
Gamma globulin (\%) & $0.19 \pm 0.07$ \\
\hline GOT: glutamic-Oxaloacetic & \\
\hline
\end{tabular}

GOT: glutamic-oxaloacetic transaminase; GPT: glutamic-pyruvic transaminase; ALP: alkaline phosphatase; ESR: erythrocyte sedimentation rate; ANA: antinuclear antibody; AMA: antimitochondrial antibodies; ANCA: anti-neutrophil cytoplasmic antibody; ASMA: anti-smooth muscle antibody; anti-LKM: anti-liver-kidney microsomal; GGT: gamma-glutamyltransferase; CRP: C-reactive protein

Table 2. Prevalence of SARS-CoV2 antibodies in false positive serology

\begin{tabular}{lll}
\hline $\lg$ type & $\boldsymbol{N}$ & $\%$ \\
\hline $\lg \mathrm{M}^{+}$ & 6 & $42.86 \%$ \\
$\operatorname{lgG}{ }^{+}$ & 6 & $42.86 \%$ \\
$\operatorname{lgG}^{+}$and $\operatorname{lgM}^{+}$ & 2 & $14.28 \%$ \\
\hline
\end{tabular}

As the presence of autoantibodies has been associated to a high false positivity rate at viral serological tests [9], we analyzed the prevalence of autoantibodies typically involved in liver autoimmune diseases (i.e. ANA, AMA, anti-LKM antibody, ASMA, ANCA) and no significant difference was found between negative and false positive subjects (Table 3), highlighting that the cause of serological false positivity does not lie in the cross-reactivity with autoantibodies. Moreover, the presence of polyclonal hypergammaglobulinemia seems not to interfere with the serological test as well (Table 3). 
The differences between patients with negative serology and patients with false positive serology were explored. Interestingly, significantly higher levels of GGT and CRP were detected in false positive cases (Table 3). No significant differences were found in other biochemical blood tests.

Table 3. Differences between patients with false positive and negative serological test

\begin{tabular}{|c|c|c|c|}
\hline Variable & $\begin{array}{l}\text { Negative serology } \\
\text { (mean } \pm \text { SD) }\end{array}$ & $\begin{array}{l}\text { False positive serology } \\
\text { (mean } \pm S D)\end{array}$ & $P$ value \\
\hline Direct bilirubin (mg/dL) & $0.13 \pm 6.04$ & $0.25 \pm 0.26$ & 0.16 \\
\hline indirect bilirubin (mg/dL) & $0.58 \pm 0,19$ & $0.75 \pm 0,44$ & 0.25 \\
\hline GOT (U/L) & $38.46 \pm 5.8$ & $41.85 \pm 8.00$ & 0.73 \\
\hline GPT (U/L) & $37.69 \pm 24,69$ & $45.57 \pm 20.65$ & 0.48 \\
\hline GGT (U/L) & $57.69 \pm 38.15$ & $140.85 \pm 82.9$ & $0.03^{*}$ \\
\hline ALP (U/L) & $107.92 \pm 43.67$ & $149.57 \pm 78.84$ & 0.16 \\
\hline CRP (mg/L) & $1.73 \pm 1.70$ & $8.67 \pm 8.96$ & $0.01^{*}$ \\
\hline $\mathrm{ESR}(\mathrm{mm} / \mathrm{h})$ & $28.46 \pm 25.49$ & $25.42 \pm 13.56$ & 0.77 \\
\hline \multirow[t]{2}{*}{ Gamma globulin (\%) } & $0.18 \pm 6.28$ & $0.21 \pm 9.46$ & 0.44 \\
\hline & $N(\%)$ & $N(\%)$ & \\
\hline $\mathrm{ANA}^{+}$ & $20(76.92 \%)$ & $8(57.14 \%)$ & n.s. \\
\hline $\mathrm{AMA}^{+}$ & $8(30.77 \%)$ & $8(57.14 \%)$ & n.s. \\
\hline Anti-LKM ${ }^{+}$ & $2(7.69 \%)$ & $0(0 \%)$ & n.s. \\
\hline $\mathrm{ASMA}^{+}$ & $8(30.76 \%)$ & $4(28.57 \%)$ & n.s. \\
\hline $\mathrm{ANCA}^{+}$ & $4(15.38 \%)$ & $0(0 \%)$ & n.s. \\
\hline Polyclonal hypergammaglobulinemia & $12(46.15 \%)$ & $6(42.86 \%)$ & n.s. \\
\hline
\end{tabular}

*Significant $P$ value; n.s.: non significant $P$ value

\section{Discussion}

Italy has been the first European country affected by COVID-19 pandemic, forcing the Government to adopt important restriction rules during the first epidemic wave and regional restrictions during the following epidemic waves. In order to guarantee an adequate hospital support to the growing demand of COVID-19 patient hospitalization, several medical Units have been converted to COVID-Units. Consequently, the follow-up of patients affected by chronic diseases was complicated by the reorganization of the health care system. In our Liver Unit several efforts were necessary to keep the health care standard quality of patients affected by chronic liver diseases, and especially of patients with liver autoimmune disorders since the potential susceptibility of these subjects to severe COVID-19. Therefore, in these patients, strict screening and monitoring were necessary by performing RT-PCR of nasopharyngeal swabs, antigenic rapid test and serological analysis during follow-up. During the observation time 2 patients (4.76\%) resulted SARS-CoV2 positive and interestingly, the serological assay generated a false positivity in 35\% of non-infected cases. Although the presence of autoantibodies in autoimmune diseases has been associated to false positive viral serologies, we did not report any correlation between the false positivity and the presence of autoantibodies. Accordingly, a cross-reactivity between autoantibodies in different autoimmune diseases and SARS-CoV2 antibodies was excluded [10], in contrast with studies conducted on SARS-CoV [11]. Moreover, Wang et al. [12] showed the presence of SARS-CoV2 IgM in $61.1 \%$ of rheumatoid factor IgM-positive sera. The presence of ANCA in patients with granulomatosis with polyangiitis (GPA) was associated with false positive SARS-CoV2 serology [13]. On the other side, it was also described that elevation of SARS-CoV2 antibodies causes a false positivity for ANA and anti-extractable nuclear antigen (ENA) due to the cross-reactivity of SARS-CoV2 antibodies with autoimmune target proteins [14].

Very interestingly, we reported that the patients with false positive serology presented significantly higher levels of GGT and CRP. We may speculate that a higher level of inflammatory state and liver damage could correlate with the serological false positivity. 
The small sample size and single centre enrolment represent study limitations. Moreover, comprehensive long-term investigation, including other types of autoimmune disorders, will be necessary to fully understand and confirm the study evidence. Although a recent study described normal outcomes in 70 COVID-19 patients with autoimmune hepatitis [15], the strategy for the SARS-CoV2 monitoring in these patients needs to be discussed and improved. Moreover, last December 2020 the vaccination campaign against COVID-19 started throughout the European Union, opening a new period for the development of surveillance studies on the efficacy and duration of protection generated by vaccines. Here, we describe a low specificity of serological analysis in subjects with liver autoimmune disorders. This becomes relevant in terms of health care decision making and studying of vaccine responses and should be considered by clinicians until new specific tests will be properly verified in this patient category.

\section{Abbreviations}

AIH: autoimmune hepatitis

AMA: antimitochondrial antibodies

ANA: antinuclear antibody

ANCA: anti-neutrophil cytoplasmic antibody

anti-LKM: anti-liver-kidney microsomal

ASMA: anti-smooth muscle antibody

COVID-19: coronavirus disease 2019

CRP: C-reactive protein

GGT: gamma-glutamyltransferase

Ig: immunoglobulin

PBC: primary biliary cholangitis

RT-PCR: reverse transcription-polymerase chain reaction

SARS-CoV2: severe acute respiratory syndrome coronavirus 2

\section{Declarations}

\section{Author contributions}

MGC contributed to data collection, study design and paper draft; MS performed the analysis and contributed to study design and paper draft; RV, FC, GDG and PC organized the database and revised sections of the manuscript; GS conceptualized and supervised the entire work.

\section{Conflicts of interest}

The authors declare that they have no conflicts of interest.

\section{Ethical approval}

All the procedures performed in this study involving human participants were in accordance with the ethical standards of the institutional and/or national research committee and with the 1964 Helsinki Declaration and its later amendments or comparable ethical standards.

\section{Consent to participate}

Informed consent to participate in the study was obtained from all participants.

\section{Consent to publication}

Not applicable.

\section{Availability of data and materials}

All relevant data is contained within the manuscript. 


\section{Funding}

Not applicable.

\section{Copyright}

(c) The Author(s) 2021.

\section{References}

1. WHO Coronavirus (COVID-19) Dashboard [Internet]. World Health Organization. c2021 [cited 2021 Jun 11]. Available from: https://covid19.who.int/

2. Grant MC, Geoghegan L, Arbyn M, Mohammed Z, McGuinness L, Clarke E, et al. The prevalence of symptoms in 24,410 adults infected by the novel coronavirus (SARS-CoV-2; COVID-19): a systematic review and meta-analysis of 148 studies from 9 countries. PloS One. 2020;15:e0234765

3. Yüce M, Filiztekin E, Özkaya KG. COVID-19 diagnosis-a review of current methods. Biosens Bioelectron. 2021;172:112752.

4. Baumgarth N, Nikolich-Žugich J,Eun-Hyung Lee F, Bhattacharya D. Antibody responses to SARS-CoV-2: let's stick to known knowns. J Immunol. 2020;205:2342-50.

5. Winthrop KL, Chiller T. Preventing and treating biologic-associated opportunistic infections. Nat Rev Rheumatol. 2009;5:405-10.

6. Dubin PJ, Kolls JK. Th17 cytokines and mucosal immunity. Immunol Rev. 2008;226:160-71.

7. Whitman JD, Hiatt J, Mowery CT, Shy BR, Yu R, Yamamoto TN, et al. Evaluation of SARS-CoV-2 serology assays reveals a range of test performance. Nat Biotechnol. 2020;38:1174-83.

8. Liberal R, Grant CR. Cirrhosis and autoimmune liver disease: current understanding. World J Hepatol. 2016;8:1157-68.

9. Kharlamova N, Dunn N, Bedri SK, Jerling S, Almgren M, Faustini F, et al. False positive results in SARS-CoV-2 serological tests for samples from patients with chronic inflammatory diseases. Front Immunol. 2021;12:666114.

10. Teng J, Dai J, Su Y, Zhou Z, Chi H, Wan L, et al. Detection of IgM and IgG antibodies against SARS-CoV-2 in patients with autoimmune diseases. Lancet Rheumatol. 2020;2:e384-5.

11. Wang Y, Sun S, Shen H, Jiang L, Zhang M, Xiao D, et al. Cross-reaction of SARS-CoV antigen with autoantibodies in autoimmune diseases. Cell Mol Immunol. 2004;1:304-7.

12. Wang $\mathrm{Q}, \mathrm{Du} \mathrm{Q}$, Guo B, Mu D, Lu X, Ma $\mathrm{Q}$, et al. A method to prevent SARS-CoV-2 IgM false positives in gold immunochromatography and enzyme-linked immunosorbent assays. J Clin. Microbiol. 2020;58:e00375-20.

13. Zouvelekis A, Karampitsakos T, Krompa A, Markozannes E, Bouros D. False positive COVID-19 antibody test in a case of granulomatosis with polyangiitis. Front Med (Lausanne). 2020;7:399.

14. Vojdani A, Kharrazian D. Potential antigenic cross-reactivity between SARS-CoV-2 and human tissue with a possible link to an increase in autoimmune diseases. Clin Immunol. 2020;217:108480.

15. Marjot T, Buescher G, Sebode M, Barnes E, Sidney Barritt 4th A, Armstrong MJ, et al. SARS-CoV-2 infection in patients with autoimmune hepatitis. J Hepatol. 2021;74:1335-43. 